\title{
Products of open manifolds with $\mathbb{R}$
}

\author{
by
}

\author{
Craig R. Guilbault (Milwaukee, WI)
}

\begin{abstract}
We present a characterization of those open $n$-manifolds $(n \geq 5)$ whose products with the real line are homeomorphic to interiors of compact $(n+1)$-manifolds with boundary.
\end{abstract}

1. Introduction. One often wishes to know whether a given open manifold can be compactified by the addition of a manifold boundary. In other words, for an open manifold $M^{n}$, we ask if there exists a compact manifold $C^{n}$ with $\operatorname{int}\left(C^{n}\right) \approx M^{n}$. Since $\operatorname{int}\left(C^{n}\right) \hookrightarrow C^{n}$ is a homotopy equivalence, and because every compact manifold has the homotopy type of a finite $\mathrm{CW}$ complex (see $[\mathrm{KS}]$ ), a necessary condition is that $M^{n}$ have finite homotopy type. This condition is not sufficient. One of the most striking illustrations of that fact occurs in a famous contractible (thus, homotopy equivalent to a point) 3-manifold constructed by J. H. C. Whitehead [Wh]. That example is best known for not being homeomorphic to $\mathbb{R}^{3}$ (or, equivalently, to $\operatorname{int}\left(B^{3}\right)$ ), but a little additional thought reveals that it is not homeomorphic to the interior of any compact 3-manifold.

Somewhat surprisingly, the product of the Whitehead manifold with a line is homeomorphic to $\mathbb{R}^{4}$. In fact, it is now known that the product of any contractible $n$-manifold with a line is homeomorphic to $\mathbb{R}^{n+1}$. That fact was obtained through the combined efforts of several researchers; see, for example, [Gl], [Mc], [St], [Lu1], [Lu2] and [Fr]. In this note we prove the following generalization of that result:

Theorem 1.1. For an open manifold $M^{n}(n \geq 5), M^{n} \times \mathbb{R}$ is homeomorphic to the interior of a compact $(n+1)$-manifold with boundary if and only if $M^{n}$ has the homotopy type of a finite complex.

I wish to acknowledge Igor Belegradek for motivating this work by asking me the question:

2000 Mathematics Subject Classification: Primary 57N15, 57Q12.

Key words and phrases: manifold, end, stabilization, Siebenmann's thesis. 
If $M^{n}$ is an open manifold homotopy equivalent to an embedded compact submanifold, say a torus, must $M^{n} \times \mathbb{R}$ be homeomorphic to the interior of a compact manifold?

Initially, I was surprised that the question was open. The fairly obvious approach - application of the main result of Siebenmann's thesis-works nicely for $M^{n} \times \mathbb{R}^{2}$. In fact, Siebenmann himself addressed that situation in his thesis [Si1, Th. 6.12], where a key ingredient was the straightforward observation that, for any connected open manifold $M^{n}, M^{n} \times \mathbb{R}^{2}$ has stable fundamental group at infinity. We too obtain our result by applying Siebenmann's thesis; but unlike the "cross $\mathbb{R}^{2}$ situation", stability of the fundamental group at infinity for $M^{n} \times \mathbb{R}$ is not so easy. In fact, there exist open manifolds $M^{n}$ for which $M^{n} \times \mathbb{R}$ fails to have stable fundamental group at infinity. An example of that phenomenon will be provided in Section 3. However, under the (already necessary) hypothesis of finite homotopy type - or even a weaker hypothesis of finite domination - we are able to obtain $\pi_{1}$-stability in $M^{n} \times \mathbb{R}$. That is the main step in our proof. A key ingredient is the adaptation of a recent technique from [GuTi].

2. Definitions and background. Throughout this paper, we work in the PL category. Proofs can be modified in the usual ways to obtain equivalent results in the smooth or topological categories.

2.1. Neighborhoods of infinity, ends, and finite dominations. A manifold $M^{n}$ is open if it is noncompact and has no boundary. A subset $N$ of $M^{n}$ is a neighborhood of infinity if $\overline{M^{n}-N}$ is compact. We say that $M^{n}$ is oneended if each neighborhood of infinity contains a connected neighborhood of infinity; in other words, $M^{n}$ contains "arbitrarily small" connected neighborhoods of infinity. More generally, $M^{n}$ is $k$-ended $(k \in \mathbb{N})$ if it contains arbitrarily small neighborhoods of infinity consisting of exactly $k$ components, each of which has noncompact closure. If no such $k$ exists, we say $M^{n}$ has infinitely many ends.

A neighborhood of infinity is clean if it is a closed subset of $M^{n}$ and a codimension 0 submanifold with a boundary that is bicollared in $M^{n}$. By discarding compact components and drilling our neighborhoods arcs, we can find, within any clean neighborhood of infinity $N$, an improved clean neighborhood of infinity $N^{\prime}$ having the properties:

- $N^{\prime}$ contains no compact components,

- each component of $N^{\prime}$ has connected boundary.

If $M^{n}$ is $k$-ended, then there exist arbitrarily small improved neighborhoods of infinity containing exactly $k$ components. Such a neighborhood is called 
a 0-neighborhood of infinity. In this situation, we may choose a sequence

$$
N_{1} \supseteq N_{2} \supseteq N_{3} \supseteq \cdots
$$

of 0-neighborhoods of infinity such that $N_{i+1} \subseteq \operatorname{int}\left(N_{i}\right)$ for all $i$ and $\bigcap_{i=1}^{\infty} N_{i}$ $=\emptyset$. A sequence of this sort will be referred to as neat. Then for each $i$, the components may be indexed as $N_{i}^{1}, \ldots, N_{i}^{k}$; furthermore, these indices may be chosen coherently so that for all $i<i^{\prime}$ and all $1 \leq j \leq k$ we have $N_{i^{\prime}}^{j} \subseteq \operatorname{int}\left(N_{i}^{j}\right)$. When all of the above has been accomplished, we will refer to $\left\{N_{i}\right\}_{i=1}^{\infty}$ as a well-indexed neat sequence of 0-neighborhoods of infinity. For a fixed $j$, we say that the nested sequence $\left\{N_{i}^{j}\right\}_{i=1}^{\infty}$ of components represents the $j$ th end of $M^{n}$.

A space has finite homotopy type if it is homotopy equivalent to a finite CW complex. A space $X$ is finitely dominated if there exists a finite complex $L$ and maps $u: X \rightarrow L$ and $d: L \rightarrow X$ such that $d \circ u \simeq \mathrm{id}_{X}$. It is a standard fact that a polyhedron (or complex) $X$ is finitely dominated if and only if there is a homotopy $H: X \times[0,1] \rightarrow X$ such that $H_{0}=\operatorname{id}_{X}$ and $\overline{H_{1}(X)}$ is compact. (We say $H$ pulls $X$ into a compact set.) For later use, we prove a mild refinement of this latter characterization.

Lemma 2.1. A polyhedron $X$ is finitely dominated if and only if, for any compactum $C \subseteq X$, there is a homotopy $J: X \times[0,1] \rightarrow X$ such that

(i) $J_{0}=\mathrm{id}_{X}$,

(ii) $\overline{J_{1}(X)}$ is compact,

(iii) $\left.J\right|_{C \times[0,1]}=\operatorname{id}_{C \times[0,1]}$.

Proof. We need only prove the forward implication, as the converse is obvious. Begin with a homotopy $H: X \times[0,1] \rightarrow X$ satisfying the analogues of conditions (i) and (ii). Choose a compact polyhedral neighborhood $D$ of $C$ in $X$. Then define $J$ on the union of $X \times\{0\}$ and $(C \cup(X-\operatorname{int}(D))) \times[0,1]$ as follows:

$$
J(x, t)= \begin{cases}x & \text { if } t=0, \\ x & \text { if } x \in C, \\ H(x, t) & \text { if } x \in X-\operatorname{int} D .\end{cases}
$$

Apply the homotopy extension theorem [Hu, §IV.2] to extend $J$ to all of $X \times[0,1]$. Condition (ii) follows from compactness of $D$.

If a space is finitely dominated, one often wishes to know whether it has finite homotopy type. This issue was resolved by Wall in [Wa] where, for every finitely dominated $X$, there is defined an obstruction $\sigma(X)$ lying in the reduced projective class group $\widetilde{K}_{0}\left(\mathbb{Z}\left[\pi_{1}(X)\right]\right)$. This obstruction vanishes if and only if $X$ has finite homotopy type.

A space having finite homotopy type may have infinitely many ends. One example is the universal cover of a figure-eight. However, within the realm 
of open manifolds, this does not happen. Indeed, a straightforward duality argument provides the following bound. (See, for example [Si2].) We provide a slightly more geometric argument.

LEMma 2.2. The number of ends of a finitely dominated open n-manifold $M^{n}$ is a finite integer bounded above by $\operatorname{rank}\left(H_{n-1}\left(M^{n}, \mathbb{Z}_{2}\right)\right)+1$.

Sketch of proof. If $M^{n}$ is dominated by a finite complex $L$, then each homology group of $L$ surjects onto the corresponding homology group of $M^{n}$. It follows that $\operatorname{rank}\left(H_{n-1}\left(M^{n}, \mathbb{Z}_{2}\right)\right)<\infty$. Next observe that, for an improved neighborhood of infinity $N$, the collection of boundary components of $N$ forms a nearly independent collection of elements of $H_{n-1}\left(M^{n}, \mathbb{Z}_{2}\right)$. (This is where we use the fact that $M^{n}$ is an open manifold.) So if $M^{n}$ contained improved neighborhoods of infinity with arbitrarily large numbers of components, $H_{n-1}\left(M^{n}, \mathbb{Z}_{2}\right)$ would be infinitely generated. See [GuTi, Prop. 3.1] for details.

2.2. Fundamental group at infinity and Siebenmann's thesis. For an inverse sequence

$$
G_{0} \stackrel{\lambda_{1}}{\longleftarrow} G_{1} \stackrel{\lambda_{2}}{\longleftarrow} G_{2} \stackrel{\lambda_{3}}{\longleftarrow} \cdots
$$

of groups and homomorphisms, a subsequence of $\left\{G_{i}, \lambda_{i}\right\}$ is an inverse sequence of the form

$$
G_{i_{0}} \stackrel{\phi_{1}}{\longleftarrow} G_{i_{1}} \stackrel{\phi_{2}}{\longleftarrow} G_{i_{2}} \stackrel{\phi_{3}}{\longleftarrow} \cdots,
$$

where, for each $j$, the homomorphism $\phi_{j}$ is the obvious composition $\lambda_{i_{j-1}+1}$ ○ $\cdots \circ \lambda_{i_{j}}$ of homomorphisms from the original sequence. We say that $\left\{G_{i}, \lambda_{i}\right\}$ is stable if it contains a subsequence $\left\{G_{i_{j}}, \phi_{j}\right\}$ that induces a sequence of isomorphisms

$$
\operatorname{im}\left(\phi_{1}\right) \cong \operatorname{im}\left(\phi_{2}\right) \cong \operatorname{im}\left(\phi_{3}\right) \cong \ldots .
$$

If a sequence $(*)$ exists where the bonding maps are simply injections, we say that $\left\{G_{i}, \lambda_{i}\right\}$ is pro-injective; if one exists where the bonding maps are surjections, we say that $\left\{G_{i}, \lambda_{i}\right\}$ is pro-surjective or (more commonly) semistable.

For a one-ended open manifold $M^{n}$ and a neat sequence $\left\{N_{i}\right\}_{i=1}^{\infty}$ of 0-neighborhoods of infinity, choose basepoints $p_{i} \in N_{i}$, and paths $\alpha_{i} \subset N_{i}$ connecting $p_{i}$ to $p_{i+1}$. Then construct an inverse sequence of groups:

$$
\pi_{1}\left(N_{0}, p_{0}\right) \stackrel{\lambda_{1}}{\longleftarrow} \pi_{1}\left(N_{1}, p_{1}\right) \stackrel{\lambda_{2}}{\longleftarrow} \pi_{1}\left(N_{2}, p_{2}\right) \stackrel{\lambda_{3}}{\longleftarrow} \cdots
$$

by letting $\lambda_{i+1}: \pi_{1}\left(N_{i+1}, p_{i+1}\right) \rightarrow \pi_{1}\left(N_{i}, p_{i}\right)$ be the homomorphism induced by inclusion followed by the change of basepoint isomorphism determined by $\alpha_{i}$. The obvious singular ray obtained by piecing together the $\alpha_{i}$ 's is often referred to as the base ray for this inverse sequence. This inverse sequence (or more precisely the "pro-equivalence class" of this sequence) is referred to as the fundamental group at infinity for $M^{n}$ and is denoted by $\pi_{1}\left(\varepsilon\left(M^{n}\right)\right)$. 
REMARK 1. For the purposes of this paper, we only need to consider the fundamental group at infinity for one-ended manifolds. (Even though we often begin with a multi-ended manifold.) In multi-ended situations, one may associate a different inverse sequence to each end. For example, if $M^{n}$ is a $k$-ended open manifold and $\left\{N_{i}\right\}_{i=1}^{\infty}$ is a well-indexed neat sequence of 0 -neighborhoods of infinity, then for each $j \in\{1, \ldots, k\}$ we can construct an inverse sequence

$$
\pi_{1}\left(N_{0}^{j}\right) \stackrel{\lambda_{1}^{j}}{\longleftarrow} \pi_{1}\left(N_{1}^{j}\right) \stackrel{\lambda_{2}^{j}}{\longleftarrow} \pi_{1}\left(N_{2}^{j}\right) \stackrel{\lambda_{3}^{j}}{\longleftarrow} \cdots,
$$

which is called the fundamental group at the $j$ th end of $M^{n}$. (Here we have omitted reference to basepoints only to simplify notation.)

For a more thorough discussion of inverse sequences and the fundamental group system at infinity, see [Gu].

As indicated in the introduction, Theorem 1.1 will be obtained as a consequence of the main result of [Si1]. For easy reference, we state that result and provide some necessary definitions.

Theorem 2.3 (Siebenmann, 1965). A one-ended open $n$-manifold $M^{n}$ $(n \geq 6)$ is homeomorphic to the interior of a compact manifold with boundary iff:

(1) $M^{n}$ is inward tame at infinity,

(2) $\pi_{1}$ is stable at infinity,

(3) $\sigma_{\infty}\left(M^{n}\right) \in \widetilde{K}_{0}\left(\mathbb{Z}\left[\pi_{1}\left(\varepsilon\left(M^{n}\right)\right)\right]\right)$ is trivial.

In the above, inward tame at infinity (or simply "inward tame") means that for any neighborhood $N$ of infinity, there exists a homotopy (sometimes called a taming homotopy) $H: N \times[0,1] \rightarrow V$ such that $H_{0}=$ id and $\overline{H_{1}(N)}$ is compact. Equivalently, $M^{n}$ is inward tame if all clean neighborhoods of infinity are finitely dominated. If $N \supseteq N^{\prime}$ are clean neighborhoods of infinity, then any taming homotopy for $N^{\prime}$ can be extended to a taming homotopy for $N$. Thus, in order to prove inward tameness for $M^{n}$, it suffices to show the existence of arbitrarily small finitely dominated clean neighborhoods of infinity.

Given conditions (1) and (2) above, one may choose a 0-neighborhood of infinity $N$ with the "correct" fundamental group - as determined by (2). Then $\sigma_{\infty}\left(M^{n}\right)$ is the Wall finiteness obstruction of $N$. With some additional work, one sees that $\sigma_{\infty}\left(M^{n}\right)$ is trivial if and only if all clean neighborhoods of infinity in $M^{n}$ (or equivalently, arbitrarily small clean neighborhoods of infinity) have finite homotopy type. For more details see [Si1] or [Gu].

REMARK 2. By giving a more general definition of $\sigma_{\infty}\left(M^{n}\right)$, it is possible to separate conditions (2) and (3); this has been done in $[\mathrm{Gu}]$. However, in 
the current context, it seems better to keep Theorem 2.3 in its traditional form.

2.3. Combinatorial group theory and the generalized Seifert-van Kampen theorem. The last bit of background information we wish to comment on is primarily combinatorial group theory. Given groups $G_{0}, G_{1}$ and $G_{2}$ and homomorphisms $i_{1}: G_{0} \rightarrow G_{1}$ and $i_{2}: G_{0} \rightarrow G_{2}$ we call $G$ the pushout of $\left(i_{1}, i_{2}\right)$ if there exist homomorphisms $j_{1}$ and $j_{2}$ completing the commutative diagram

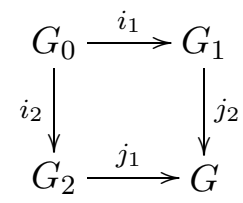

and satisfying the following "universal mapping property":

If homomorphisms $k_{1}: G_{1} \rightarrow H$ and $k_{2}: G_{2} \rightarrow H$ allow for a similar commutative diagram, then there exists a unique homomorphism $\varphi$ :

$G \rightarrow H$ such that $j_{1} \circ \varphi=k_{1}$ and $j_{2} \circ \varphi=k_{2}$.

In this case, $G$ is uniquely determined up to isomorphism.

In the special case where the above homomorphisms $i_{1}$ and $i_{2}$ are injective, the pushout is called a free product with amalgamation of $G_{1}$ and $G_{2}$ along $G_{0}$ and is denoted by $G_{1} *_{G_{0}} G_{2}$ With this terminology, we are implicitly viewing $G_{0}$ as a subgroup of both $G_{1}$ and $G_{2}$. Then $G_{1} *_{G_{0}} G_{2}$ is the result of "gluing" $G_{1}$ to $G_{2}$ along $G_{0}$. More precisely, if $\left\langle A_{1} \mid R_{1}\right\rangle$ and $\left\langle A_{1} \mid R_{1}\right\rangle$ are presentations for $G_{1}$ and $G_{2}$ and $B$ is a generating set for $G_{0}$, then $\left\langle A_{1} \cup A_{2} \mid R_{1}, R_{2}, S\right\rangle$ is a presentation for $G_{1} *_{G_{0}} G_{2}$ where

$$
S=\left\{i_{1}(y)^{-1} i_{2}(y) \mid y \in B\right\} .
$$

(The same procedure produces presentations for arbitrary pushouts.)

In topology, the most common application of "pushout diagrams" is found in the Seifert-van Kampen theorem [Ma, Ch. 4], which may be stated as follows: if a space $X$ is expressed as a union $X=U \cup V$ of path connected open sets such that $U \cap V$ is also path connected and $x \in U \cap V$, then $\pi_{1}(X, x)$ is the pushout of

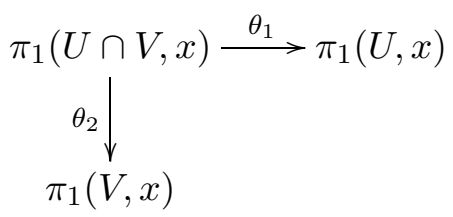

where $\theta_{1}$ and $\theta_{2}$ are induced by inclusion. In most cases, $\theta_{1}$ and $\theta_{1}$ are not injective, so $\pi_{1}(X, x)$ is not necessarily a free product with amalgamation. 
The above group-theoretic constructions can be extended to generalized graphs of groups and the more restrictive graphs of groups. For either of these constructions, we begin with an oriented graph $\Delta$. (Here a "graph" is simply a 1-dimensional CW complex.) Then to each vertex $v$ we associate a "vertex group" $G_{v}$ and to each edge $e$ we associate an "edge group" $G_{e}$. In addition, for each edge group $G_{e}$ we need "edge homomorphisms" $\varphi_{e}^{+}$and $\varphi_{e}^{-}$mapping $G_{e}$ into the vertex group at the "positive" and "negative" end of $e$, respectively. (If $e$ is a loop in $\Delta$, then $\varphi_{e}^{+}$and $\varphi_{e}^{-}$can be different homomorphisms into the same vertex group.) Let $(\mathcal{G}, \Delta)$ represent this setup. If each edge homomorphism is injective, call $(\mathcal{G}, \Delta)$ a "graph of groups"; otherwise it is just a "generalized graph of groups".

Our next task is to assign, to an arbitrary generalized graph of groups $(\mathcal{G}, \Delta)$, a single group that generalizes the pushout construction for the simple case. This could be done with a universal mapping property. Instead, we describe a specific construction of the group. Let $V$ [resp., $E]$ denote the collection of vertices [resp., edges] of $\Delta$. Choose a maximal tree $\Upsilon$ in $\Delta$. Then the fundamental group of $(\mathcal{G}, \Delta)$ based at $\Upsilon$ is the group

$$
\pi_{1}(\mathcal{G}, \Delta ; \Upsilon)=\left(\left(\underset{v \in V}{*} G_{v}\right) * F_{E}\right) / N
$$

where $*_{v \in V} G_{v}$ is the free product of all vertex groups, $F_{E}$ is the free group generated by the set $E$, and $N$ is the smallest normal subgroup of $\left(*_{v \in V} G_{v}\right)$ $* F_{E}$ generated by the set

$$
\left\{e^{-1} \cdot \varphi_{e}^{-}(x) \cdot e \cdot\left(\varphi_{e}^{+}(x)\right)^{-1} \mid e \in E \text { and } x \in G_{e}\right\} \cup\{e \mid e \in \Upsilon\} .
$$

EXAMPLE 1. Diagram $(\diamond)$ determines a generalized graph of groups where the graph is simply an oriented interval; moreover, the fundamental group of that generalized graph of groups is precisely the pushout of that diagram. When $i_{1}$ and $i_{2}$ are injective we have a genuine graph of groups whose fundamental group is a free product with amalgamation.

A similar special case - this time, a graph of groups with just one vertex and one edge - leads to another well-known construction in combinatorial group theory: the HNN extension.

See $[\mathrm{Co}]$ for details on the above ideas.

In group theory, it is preferable to study free products with amalgamation over arbitrary pushouts. Similarly, genuine graphs of groups are preferable to generalized graphs of groups. However, as noted above, arbitrary pushouts occur naturally in topology via the classical Seifert-van Kampen theorem. Similarly, the following generalized Seifert-van Kampen theorem frequently leads to a generalized graph of groups.

Theorem 2.4 (Generalized Seifert-van Kampen). Suppose a path connected space $X$ may be expressed as a union of path connected open subsets 
$\left\{U_{\alpha}\right\}_{\alpha \in A}$ so that no point of $X$ lies in more than two of the $U_{\alpha}$ 's. Let $\Delta$ be the graph having vertex set $A$, and one edge between $U_{\alpha}$ and $U_{\alpha^{\prime}}$ for each path component $V_{\alpha \alpha^{\prime} \beta}$ of $U_{\alpha} \cap U_{\alpha^{\prime}}\left(\alpha \neq \alpha^{\prime} \in A\right)$. Place an arbitrarily chosen orientation on each edge; then choose a basepoint from each $U_{\alpha}[$ resp., each $\left.V_{\alpha \alpha^{\prime} \beta}\right]$ and associate to the corresponding vertex [resp., edge] the fundamental group of $U_{\alpha}\left[r e s p\right.$., $\left.V_{\alpha \alpha^{\prime} \beta}\right]$. For each $V_{\alpha \alpha^{\prime} \beta}$, choose paths in $U_{\alpha}$ and $U_{\alpha^{\prime}}$ respectively, connecting the basepoint of $V_{\alpha \alpha^{\prime} \beta}$ to the basepoints of $U_{\alpha}$ and $U_{\alpha^{\prime}}$. Let the two edge homomorphisms for $V_{\alpha \alpha^{\prime} \beta}$ be those induced by inclusion followed by change of basepoints along these paths. If $(\mathcal{G}, \Delta)$ denotes this graph of groups, then $\pi_{1}(X, x)$ is isomorphic to $\pi_{1}(\mathcal{G}, \Delta ; \Upsilon)$ where $\Upsilon$ is an arbitrarily chosen maximal tree in $\Delta$.

Proof. See Chapter 2 of [Ge].

3. Proof of Theorem 1.1. In order to prove Theorem 1.1 we need only show that, if an open manifold $M^{n}$ has finite homotopy type, then $M^{n} \times \mathbb{R}$ is a one-ended open manifold satisfying all three conditions of Theorem 2.3. Proposition 3.1 begins that process; part (a) asserts that $M^{n} \times \mathbb{R}$ is one-ended and open, while part (b) ensures that condition (1) holds. Strictly speaking, the "end obstruction" $\sigma_{\infty}\left(M^{n} \times \mathbb{R}\right)$ found in Theorem 2.3 cannot be defined until it is known that condition (2) holds. Even so, it is possible to address condition (3) before condition (2) by proving that all clean neighborhoods of infinity in $M^{n} \times \mathbb{R}$ have finite homotopy type. This will be done (under the assumption that $M^{n}$ has finite homotopy type) in part (c). Therefore, in the context of Theorem 1.1, once condition (2) is verified, condition (3) follows immediately. To simplify the discussion, we refer to a manifold in which all clean neighborhoods of infinity have finite homotopy type as super-inward tame at infinity.

3.1. Conditions (1) and (3) of Theorem 2.3. Before stating Proposition 3.1 , we introduce some terminology and notation to be used throughout the rest of this paper. Given a connected open manifold $M^{n}$, a clean neighborhood of infinity $N \subseteq M^{n}$, and $m>0$, the associated neighborhood of infinity in $M^{n} \times \mathbb{R}$ is the set

$$
W(N, m)=(N \times \mathbb{R}) \cup\left(M^{n} \times((-\infty,-m] \cup[m, \infty))\right)
$$

It is easy to see that $W(N, m)$ is indeed a neighborhood of infinity, that $W(N, m)$ is always connected, and that $W(N, m)$ is a 0 -neighborhood of infinity in $M^{n} \times \mathbb{R}$ whenever $N$ is a 0-neighborhood of infinity in $M^{n}$.

In addition, let

$$
\begin{aligned}
& W^{+}(N, m)=(N \times \mathbb{R}) \cup\left(M^{n} \times[m, \infty)\right), \\
& W^{-}(N, m)=(N \times \mathbb{R}) \cup\left(M^{n} \times(-\infty,-m]\right) .
\end{aligned}
$$


Then $W^{+}(N, m)$ deformation retracts onto $M^{n} \times\{m\}$ and $W^{-}(N, m)$ deformation retracts onto $M^{n} \times\{-m\}$, so both are homotopy equivalent to $M^{n}$. Moreover, $W^{+}(N, m) \cap W^{-}(N, m)=N \times \mathbb{R} \simeq N$.

Proposition 3.1. Let $M^{n}$ be a connected open n-manifold. Then:

(a) $M^{n} \times \mathbb{R}$ is a one-ended open $(n+1)$-manifold.

(b) $M^{n} \times \mathbb{R}$ is inward tame at infinity iff $M^{n}$ is finitely dominated.

(c) $M^{n} \times \mathbb{R}$ is super-inward tame at infinity iff $M^{n}$ has finite homotopy type.

Proof. As noted above, all neighborhoods of infinity of the type $W(N, m)$ are connected; moreover, they can be made arbitrarily small by choosing $N$ to be small and $m$ large. Therefore, $M^{n} \times \mathbb{R}$ is one-ended.

The forward implications of assertions (b) and (c) are immediate. In particular, since $M^{n} \times \mathbb{R}$ itself is a clean neighborhood of infinity in $M^{n} \times \mathbb{R}$, both implications can be deduced from the homotopy equivalence $M^{n} \simeq$ $M^{n} \times \mathbb{R}$. Thus, we turn our attention to the two converses.

Given $W(N, m)$, let $C=M^{n}-\operatorname{int}(N)$ (a compact codimension 0 submanifold of $M^{n}$ ). Then let $C^{\prime}$ denote a second "copy" of $C$ disjoint from $M^{n}$, and $K_{N}$ be the adjunction space

$$
K_{N}=M^{n} \cup_{\varphi} C^{\prime}
$$

obtained by attaching $C^{\prime}$ to $M^{n}$ along its boundary via the "identity map" $\varphi: \partial C^{\prime} \rightarrow \partial C$.

It is easy to see that $K_{N}$ is homotopy equivalent to $W(N, m)$; indeed, $W(N, m)$ deformation retracts onto the subset

$$
\left(M^{n} \times\{m\}\right) \cup(\partial C \times[-m, m]) \cup(C \times\{-m\}),
$$

which is homeomorphic to $K_{N}$. Thus, to show that $M^{n} \times \mathbb{R}$ is inward tame at $\infty$ [resp., $M^{n} \times \mathbb{R}$ is super-inward tame at $\infty$ ], it suffices to show that $K_{N}$ is finitely dominated [resp., has $K_{N}$ finite homotopy type].

Claim 1. If $M^{n}$ is finitely dominated, then $K_{N}$ is finitely dominated.

By Lemma 2.1, we may choose a homotopy $J: M^{n} \times[0,1] \rightarrow M^{n}$ such that $\left.J\right|_{\left(M^{n} \times\{0\}\right) \cup(C \times[0,1])}$ is the identity, and $\overline{J_{1}\left(M^{n}\right)}$ is compact. Extend $J$ to a homotopy $J^{*}: K_{N} \times[0,1] \rightarrow K_{N}$ by letting $J^{*}$ be the identity over $C^{\prime}$. Then $J_{0}^{*}$ is the identity, and $J_{1}^{*}\left(K_{N}\right)$ has compact closure; so $K_{N}$ is finitely dominated.

Claim 2. If $M^{n}$ has finite homotopy type, then $K_{N}$ has finite homotopy type.

Let $f: M^{n} \rightarrow L$ be a homotopy equivalence, where $L$ is a finite complex. Then $K_{N}=M^{n} \cup_{\varphi} C^{\prime}$ is homotopy equivalent to the adjunction space

$$
L \cup_{f \circ \varphi} C^{\prime}
$$


where $f \circ \varphi$ maps $\partial C^{\prime}$ into $L$. This latter adjunction space is homotopy equivalent to a finite complex. In fact, if we begin with a triangulation of $M^{n}$ so that $C$ and $\partial C$ are subcomplexes and choose $f$ to be a cellular map, then $f \circ \varphi$ is also cellular and $L \cup_{f \circ \varphi} C^{\prime}$ is a finite complex.

3.2. Main step: stability of $\pi_{1}$ at infinity. The following will show that $M^{n} \times \mathbb{R}$ satisfies condition (2) of Theorem 2.3, and thus complete our proof of Theorem 1.1.

Proposition 3.2. If a connected open manifold $M^{n}$ is finitely dominated, then $M^{n} \times \mathbb{R}$ has stable fundamental group at infinity.

Let $M^{n}$ be a $k$-ended open $n$-manifold and $P$ and $Q$ be 0 -neighborhoods of infinity with $Q \subseteq \operatorname{int}(P)$. Index the components $P^{1}, \ldots, P^{k}$ of $P$ and $Q^{1}, \ldots, Q^{k}$ of $Q$ so that $Q^{j} \subseteq P^{j}$ for $j=1, \ldots, k$. For each $j$, let $A^{j}=$ $\overline{Q^{j}-P^{j}}$.

If $M^{n}$ is finitely dominated, choose $P$ sufficiently small that there is a homotopy $H: M^{n} \times[0,1] \rightarrow M^{n}$ pulling $M^{n}$ into $M^{n}-P$. In addition (by Lemma 2.1) arrange that $H$ is fixed over some nonempty open set $U$. To simplify notation, we focus on a single end; in particular, let $j \in\{1, \ldots, k\}$ be fixed. Choose basepoints $p_{*} \in U, p \in \partial P^{j}$ and $q \in \partial Q^{j}$; then choose a proper embedding $r:[0, \infty) \rightarrow M^{n}$ such that $r(0)=p_{*}, r(1)=p, r(2)=q$, and so that the image ray $R=r([0, \infty))$ intersects each of $\partial P^{j}$ and $\partial Q^{j}$ transversely once and only once at the points $p$ and $q$, respectively. Let $\alpha=R \cap A^{j}$ denote the corresponding arc in $A^{j}$ between $p$ and $q$.

Let $t: B^{n-1} \times[-1, \infty) \rightarrow M^{n}$ be a homeomorphism onto a regular neighborhood $T$ of $R$ so that $\left.t\right|_{\{\overline{0}\} \times[0, \infty)}=r$, and so that $T \cap A^{j}$ is a relative regular neighborhood of $\alpha$ in $A^{j}$ intersecting $\partial P^{j}$ and $\partial Q^{j}$ in $(n-1)$-disks $D$ and $D^{\prime}$, with $D=t\left(B^{n-1} \times\{1\}\right)$ and $D^{\prime}=t\left(B^{n-1} \times\{2\}\right)$. Then choose an $(n-1)$-ball $B_{0} \subseteq \operatorname{int}\left(B^{n-1}\right)$, centered at $\overline{0}$; and let $T_{0}=t\left(B_{0} \times[-1, \infty)\right)$ be the corresponding smaller regular neighborhood of $R$, with corresponding subdisks $D_{0}$ and $D_{0}^{\prime}$ contained $\operatorname{in} \operatorname{int}(D)$ and $\operatorname{int}\left(D^{\prime}\right)$, respectively. We now utilize the "homotopy refinement procedure" developed on pages 267-268 of [GuTi] to replace $H$ with a new homotopy $K: M^{n} \times[0,1] \rightarrow M^{n}$ which, in addition to pulling $M^{n}$ into $M^{n}-P$, has the properties:

(i) $K$ is "canonical" over $T_{0}$,

(ii) tracks of points lying outside $T_{0}$ do not pass through the interior of $T_{0}$.

The first of these properties arranges that all tracks of points in $R$ proceed monotonically in $R$ to $p_{*}$; and that $\left.K\right|_{D_{0}^{\prime} \times[0,1]}$ takes $D_{0}^{\prime} \times[0,1 / 2]$ homeomorphically onto $t\left(B_{0} \times[0,2]\right)$, with $D_{0}^{\prime} \times[1 / 2,1]$ being flattened onto $t\left(B_{0} \times\{0\}\right)$. We may also arrange that $K\left(D_{0}^{\prime} \times\{1 / 4\}\right)=D_{0}$. See $[\mathrm{GuTi}]$ for details. 
Proposition 3.3. Assume the above setup, with $M^{n}$ finitely dominated, $j \in\{1, \cdots, k\}$ fixed, and all previous notation unchanged. Then every loop $\tau$ in $P^{j}$ based at $p$ is homotopic (rel p) in $M^{n}$ to a loop of the form $\alpha * \tau^{\prime} * \alpha^{-1}$, where $\tau^{\prime}$ is a loop in $Q^{j}$ based at $q$.

Proof. Every loop in $P^{j}$ based at $p$ is homotopic (rel $p$ ) to a product $\tau_{1} * \cdots * \tau_{u}$ where, for each $v$, either $\tau_{v}$ lies entirely in $A^{j}$ or $\tau_{v}$ is (already) a loop of the form $\alpha * \tau_{v}^{\prime} * \alpha^{-1}$ where $\tau_{v}^{\prime}$ is a loop in $Q^{j}$ based at $q$. So, without loss of generality, we assume that $\tau$ lies entirely in $A^{j}$.

Consider the map $L=\left.K\right|_{\partial Q^{j} \times[0,1]}: \partial Q^{j} \times[0,1] \rightarrow M^{n}$. Choose triangulations $\Delta_{1}$ and $\Delta_{2}$ of the domain and range, respectively. Without changing its definition on $\left(\partial Q^{j} \times\{0\}\right) \cup\left(D_{0}^{\prime} \times[0,1 / 2]\right)$, adjust $L$ (up to a small homotopy) to a nondegenerate simplicial map. Then adjust $\tau$ (rel $p$ ) to an embedded circle in general position with respect to $\Delta_{2}$, lying entirely in $\operatorname{int}\left(A^{j}\right)$, except at its basepoint $p$, which lies in $\partial A^{j}$. Then $L^{-1}(\tau)$ is a closed 1-manifold lying in $\partial Q^{j} \times(0,1)$. Let $\sigma$ be the component of $L^{-1}(\tau)$ containing the point $(q, 1 / 4)$. Since $L$ takes a neighborhood of $(q, 1 / 4)$ homeomorphically onto a neighborhood of $p$, and since no other points of $\sigma$ are taken near $p$ (use property (ii) above), we see that $L$ takes $\sigma$ onto $\tau$ in a degree 1 fashion. Now the natural deformation retraction of $\partial Q \times[0,1]$ onto $\partial Q \times\{0\}$ pushes $\sigma$ into $\partial Q \times\{0\}$, while sliding $(q, 1 / 4)$ along the arc $\{q\} \times[0,1 / 4]$. Composing this push with $L$ provides a homotopy of $\tau$ to a loop $\tau^{\prime}$ in $\partial Q$, whereby the basepoint $p$ is slid along $\alpha$ to $q$. This provides the desired (basepoint preserving) homotopy from $\tau$ to $\alpha * \tau^{\prime} * \alpha^{-1}$.

Corollary 3.4. Assume the full setup for Proposition 3.3 and let

$$
\Gamma_{P^{j}}=\operatorname{im}\left(\pi_{1}\left(P^{j}, p\right) \rightarrow \pi_{1}\left(M^{n}, p\right)\right), \quad \Gamma_{Q^{j}}=\operatorname{im}\left(\pi_{1}\left(Q^{j}, q\right) \rightarrow \pi_{1}\left(M^{n}, q\right)\right) .
$$

Then the change of basepoint isomorphism $\widehat{\alpha}: \pi_{1}\left(M^{n}, q\right) \rightarrow \pi_{1}\left(M^{n}, p\right)$ takes $\Gamma_{Q^{j}}$ isomorphically onto $\Gamma_{P^{j}}$.

Proof. Since $\alpha \cup Q^{j} \subseteq P^{j}$, it is clear that $\widehat{\alpha}$ takes $\Gamma_{Q^{j}}$ into $\Gamma_{P^{j}}$. Injectivity is immediate, and Proposition 3.3 ensures surjectivity.

We now turn our attention back to the manifold $M^{n} \times \mathbb{R}$. In order to understand the fundamental group system at infinity, it will suffice to understand "special" neighborhoods of infinity of the sort $W(N, m)$ (along with corresponding bonding maps). To simplify the exposition, we first consider the special case where $M^{n}$ itself is one-ended. Afterwards we upgrade the proof so that it includes the general case.

Proposition 3.5. Suppose $M^{n}$ is a one-ended open n-manifold and $P$ and $Q$ are 0 -neighborhoods of infinity in $M^{n}$ with $Q \subseteq \operatorname{int}(P)$. Choose $p \in \partial P, q \in \partial Q$ and $\alpha$ a path in $\overline{P-Q}$ connecting $p$ to $q$. For $0<m<$ $m^{\prime}<\infty$, let $W(P, m) \supseteq W\left(Q, m^{\prime}\right)$ be corresponding neighborhoods of infinity 
in $M^{n} \times \mathbb{R}$ and $\lambda: \pi_{1}\left(W\left(Q, m^{\prime}\right),(q, 0)\right) \rightarrow \pi_{1}(W(P, m),(p, 0))$ the homomorphism induced by inclusion followed by a change of basepoints along $\alpha \times 0$. Then:

(1) $\pi_{1}(W(P, m),(p, 0)) \cong \pi_{1}\left(M^{n}, p\right) *_{\Gamma_{P}} \pi_{1}\left(M^{n}, p\right)$, where

$$
\Gamma_{P}=\operatorname{im}\left(\pi_{1}(P, p) \rightarrow \pi_{1}\left(M^{n}, p\right)\right),
$$

(2) $\pi_{1}\left(W\left(Q, m^{\prime}\right),(q, 0)\right) \cong \pi_{1}\left(M^{n}, q\right) * \Gamma_{Q} \pi_{1}\left(M^{n}, q\right)$, where

$$
\Gamma_{Q}=\operatorname{im}\left(\pi_{1}(Q, q) \rightarrow \pi_{1}\left(M^{n}, q\right)\right),
$$

(3) the homomorphism $\lambda$ is surjective,

(4) if there exists a homotopy pulling $M^{n}$ into $M^{n}-P$, then $\lambda$ is an isomorphism.

Proof. Using our earlier notation, the Seifert-van Kampen theorem establishes $\pi_{1}(W(P, m),(p, 0))$ as the pushout of the diagram

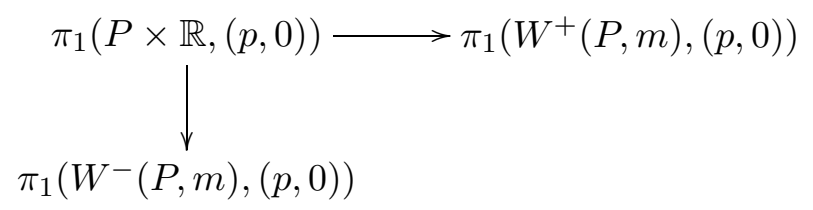

where both homomorphisms are induced by inclusion. The homotopy equivalences

$$
\begin{aligned}
(P \times \mathbb{R},(p, 0)) & \simeq(P, p), \\
\left(W^{+}(P, m),(p, 0)\right) & \simeq\left(M^{n}, p\right) \simeq\left(W^{-}(P, m),(p, 0)\right)
\end{aligned}
$$

allow us to replace the above with a simpler diagram

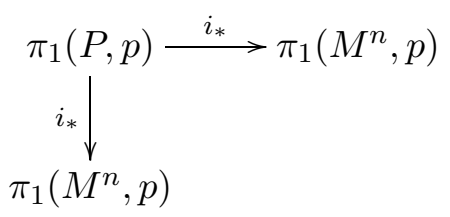

This diagram does not define a free product with amalgamation since $i_{*}$ need not be injective, however, the pushout is identical to that of

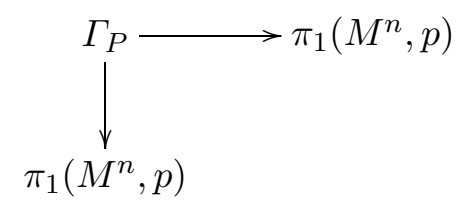

(both homomorphisms are inclusions), which determines the free product with amalgamation promised in (1).

Of course, assertion (2) is identical to (1). Then, from (\#) and the analogous diagram for $\pi_{1}\left(W\left(Q, m^{\prime}\right),(q, 0)\right)$, we see that $\lambda$ is induced by the 
isomorphism $\pi_{1}\left(M^{n}, q\right) * \pi_{1}\left(M^{n}, q\right) \rightarrow \pi_{1}\left(M^{n}, p\right) * \pi_{1}\left(M^{n}, p\right)$ by quotienting out (in the domain and range) by relations induced by $\Gamma_{Q}$ and $\Gamma_{P}$, respectively - according to the definition of free product with amalgamation. Thus, $\lambda$ is necessarily surjective. Moreover, if there is a homotopy pulling $M^{n}$ into $P$, Corollary 3.4 ensures that $\lambda$ is an isomorphism.

COROLlary 3.6. Let $M^{n}$ be a connected, one-ended open n-manifold. Then $M^{n} \times \mathbb{R}$ is a one-ended open $(n+1)$-manifold with semistable fundamental group at infinity. If $M^{n}$ is finitely dominated, then $M^{n} \times \mathbb{R}$ has stable fundamental group at infinity which is pro-isomorphic to the system $\pi_{1}\left(M^{n}\right) *_{\Gamma} \pi_{1}\left(M^{n}\right) \stackrel{\text { id }}{\longleftarrow} \pi_{1}\left(M^{n}\right) *_{\Gamma} \pi_{1}\left(M^{n}\right) \stackrel{\text { id }}{\longleftarrow} \pi_{1}\left(M^{n}\right) *_{\Gamma} \pi_{1}\left(M^{n}\right) \stackrel{\text { id }}{\longleftarrow} \cdots$, where $\Gamma$ is the image (translated by an appropriate change of basepoint isomorphism) of the fundamental group of any sufficiently small 0-neighborhood of infinity in $\pi_{1}\left(M^{n}\right)$.

Proof. This corollary is almost immediate. One simply chooses a neat sequence $\left\{N_{i}\right\}_{i=1}^{\infty}$ of 0 -neighborhoods of infinity in $M^{n}$, then applies the previous proposition (repeatedly) to the sequence $\left\{W\left(N_{i}, i\right)\right\}_{i=1}^{\infty}$. If $M^{n}$ is finitely dominated, $N_{1}$ should be chosen sufficiently small that $M^{n}$ can be pulled into $M^{n}-N_{1}$.

In the introduction, we noted that, without the hypothesis of finite domination on $M^{n}$, the fundamental group at infinity in $M^{n} \times \mathbb{R}$ need not be stable. This is now easy to exhibit.

EXAMPLE 2 (An $M^{n} \times \mathbb{R}$ with nonstable $\pi_{1}$ at infinity). Let $T_{1}=B^{n-1} \times S^{1}$ $\subseteq S^{n-1} \times S^{1}$, where $B^{n-1} \subseteq S^{n-1}$ is a tamely embedded $(n-1)$-ball. Then let $T_{2} \subseteq \operatorname{int}\left(T_{1}\right)$ be another (thinner) copy of $B^{n-1} \times S^{1}$ that winds around twice in the $S^{1}$ direction. Inside $T_{2}$ choose a third (even thinner) copy of $B^{n-1} \times S^{1}$ that winds through $T_{2}$ twice in the $S^{1}$ direction-and thus, four times through $T_{1}$ in the original $S^{1}$ direction. Continue this infinitely to get a nested sequence $T_{1} \supseteq T_{2} \supseteq \cdots$ so that $T_{\infty} \subseteq S^{n-1} \times S^{1}$ is the dyadic solenoid. Then $M^{n}=\left(S^{n-1} \times S^{1}\right)-T_{\infty}$ is a one-ended open $n$-manifold and each $N_{i}=T_{i}-T_{\infty}$ is a 0-neighborhood of infinity. Provided $n>3$, it is easy to see that $N_{i} \hookrightarrow T_{i}$ induces a $\pi_{1}$-isomorphism. Therefore, the inverse sequence

$$
\pi_{1}\left(N_{0}, p_{0}\right) \stackrel{\lambda_{1}}{\longleftarrow} \pi_{1}\left(N_{1}, p_{1}\right) \stackrel{\lambda_{2}}{\longleftarrow} \pi_{1}\left(N_{2}, p_{2}\right) \stackrel{\lambda_{3}}{\longleftarrow} \cdots
$$

is isomorphic to the sequence

$$
\mathbb{Z} \stackrel{\times 2}{\longleftarrow} \mathbb{Z} \stackrel{\times 2}{\longleftarrow} \mathbb{Z} \stackrel{\times 2}{\longleftarrow} \mathbb{Z} \stackrel{\times 2}{\longleftarrow} \cdots
$$

A more descriptive form of the above inverse sequence is

$$
\mathbb{Z} \hookleftarrow 2 \mathbb{Z} \hookleftarrow 4 \mathbb{Z} \hookleftarrow 8 \mathbb{Z} \hookleftarrow \cdots
$$


It follows that, for the corresponding sequence $\left\{W\left(N_{i}, i\right)\right\}_{i=1}^{\infty}$ of neighborhoods of infinity in $M^{n} \times \mathbb{R}$, we obtain a representation of the fundamental group at infinity for $M^{n} \times \mathbb{R}$ isomorphic to the sequence

$$
\mathbb{Z} *_{\mathbb{Z}} \mathbb{Z} \leftarrow \mathbb{Z} *_{2 \mathbb{Z}} \mathbb{Z} \leftarrow \mathbb{Z} *_{4 \mathbb{Z}} \mathbb{Z} \leftarrow \mathbb{Z} *_{8 \mathbb{Z}} \mathbb{Z} \leftarrow \cdots,
$$

where each bonding map is induced by the identity $\mathbb{Z} * \mathbb{Z} \rightarrow \mathbb{Z} * \mathbb{Z}$. Thus, each bond is surjective but not injective. It is easy to see that such a system cannot be stable.

REMARK 3. It is interesting to see that "crossing with $\mathbb{R}$ " takes examples with nonstable but pro-injective fundamental groups at infinity and produces examples with nonstable but pro-surjective (semistable) fundamental groups at infinity.

We are now prepared to address the general situation where $M^{n}$ is $k$ ended $(1 \leq k<\infty)$. For $k>1$, calculation of $\pi_{1}(W(N, m))$ is complicated by the fact that $W^{+}(N, m) \cap W^{-}(N, m)=N \times \mathbb{R}$ is not connected. In this situation, $\pi_{1}(W(N, m))$ is most effectively described using a (generalized or actual) graph of groups. In particular, let $\Theta_{k}$ denote the oriented graph consisting of two vertices $v^{+}$and $v^{-}$and $k$ oriented edges $e^{1}, \ldots, e^{k}$ each running from $v^{-}$to $v^{+}$. If $N^{1}, \ldots, N^{k}$ are the components of $N$ with basepoints $p^{1}, \ldots, p^{k}$ respectively, we associate the following groups and homomorphisms to $\Theta_{k}$ :

- $G\left(v^{+}\right)=\pi_{1}\left(W^{+}(N, m),\left(p^{1}, 0\right)\right), G\left(v^{-}\right)=\pi_{1}\left(W^{-}(N, m),\left(p^{1}, 0\right)\right)$.

- $G\left(e^{j}\right)=\pi_{1}\left(N^{j} \times \mathbb{R},\left(p^{j}, 0\right)\right)$ for each $j \in\{1, \ldots, k\}$.

- For each $j \in\{1, \ldots, k\}$ the homomorphism $\varphi_{j}^{+}: G\left(e^{j}\right) \rightarrow G\left(v^{+}\right)$is the composition

$$
\begin{aligned}
\pi_{1}\left(N^{j} \times \mathbb{R},\left(p^{j}, 0\right)\right) \stackrel{i_{*}}{\longrightarrow} \pi_{1}\left(W^{+}(N, m),\left(p^{j}, 0\right)\right) \\
\stackrel{\widehat{\beta^{j}}}{\longrightarrow} \pi_{1}\left(W^{+}(N, m),\left(p^{1}, 0\right)\right),
\end{aligned}
$$

where $\beta^{j}$ is an appropriately chosen path in $W^{+}(N, m)$ from $\left(p^{j}, 0\right)$ to $\left(p^{1}, 0\right)$.

- The homomorphisms $\varphi_{j}^{-}: G\left(e^{j}\right) \rightarrow G\left(v^{-}\right)$are defined similarly to the above, but with $\beta$ chosen to lie in $W^{-}(N, m)$.

Since $\varphi_{j}^{+}$and $\varphi_{j}^{-}$need not be injective, the above setup is just a generalized graph of groups. Let it be denoted by $\left(\mathcal{G}(N), \Theta_{k}\right)$

Note that the edge $e^{1}$, by itself, is a maximal tree in $\Theta_{k}$. By the generalized Seifert-van Kampen theorem, $\pi_{1}\left(N, p^{1}\right) \cong \pi_{1}\left(\mathcal{G}(N), \Theta_{k} ; e^{1}\right)$.

We may obtain a similar - but simpler - graph of groups as follows. Again we start with the graph $\Theta_{k}$. Motivated by the homotopy equivalences $W^{+}(N, m) \simeq M^{n} \simeq W^{-}(N, m)$, define both $G^{\prime}\left(v^{+}\right)$and $G^{\prime}\left(v^{+}\right)$to be $\pi_{1}\left(M^{n}, p^{1}\right)$. Then, in order to obtain injective edge homomorphisms, for 
each $j \in\{1, \ldots, k\}$, let

$$
G^{\prime}\left(e^{j}\right)=\Gamma_{N^{j}}=\operatorname{im}\left(\pi_{1}\left(N^{j}, p^{j}\right) \stackrel{i_{*}}{\longrightarrow} \pi_{1}\left(M^{n}, p^{j}\right) \stackrel{\widehat{\beta^{j}}}{\longrightarrow} \pi_{1}\left(M^{n}, p^{1}\right)\right),
$$

and let all edge homomorphisms be inclusions. Here $\beta^{j \prime}$ is a path in $M^{n}$ "parallel" to the path $\beta^{j}$ used above. This new graph of groups will be denoted $\left(\mathcal{G}^{\prime}(N), \Theta_{k}\right)$. It is easy to see a canonical isomorphism between $\pi_{1}\left(\mathcal{G}(N), \Theta_{k} ; e^{1}\right)$ and $\pi_{1}\left(\mathcal{G}^{\prime}(N), \Theta_{k} ; e^{1}\right)$.

We are now ready to state a more general version of Proposition 3.5, suitable for multi-ended $M^{n}$.

Proposition 3.7. Suppose $M^{n}$ is a $k$-ended open $n$-manifold and $P$ and $Q$ are 0 -neighborhoods of infinity in $M^{n}$ with components $P^{1}, \ldots, P^{k}$ and $Q^{1}, \ldots, Q^{k}$ such that $Q^{j} \subseteq \operatorname{int}\left(P^{j}\right)$ for each $j$. Choose basepoints $p^{j} \in \partial P^{j}$ and $q^{j} \in \partial Q^{j}$, paths $\alpha^{j}$ in $\overline{P^{j}-Q^{j}}$ connecting $p^{j}$ to $q^{j}$ and paths $\beta^{j}$ in $M^{n}$ connecting $q^{j}$ to $q^{1}$ for each $j \in\{1, \ldots, k\}$. For $0<m<m^{\prime}<\infty$, let $W(P, m) \supseteq W\left(Q, m^{\prime}\right)$ be corresponding neighborhoods of infinity in $M^{n} \times \mathbb{R}$, and $\lambda: \pi_{1}\left(W\left(Q, m^{\prime}\right),\left(q^{1}, 0\right)\right) \rightarrow \pi_{1}\left(W(P, m),\left(p^{1}, 0\right)\right)$ the homomorphism induced by inclusion followed by a change of basepoints along $\alpha^{1} \times 0$. Then:

(1) $\pi_{1}\left(W(P, m),\left(p^{1}, 0\right)\right) \cong \pi_{1}\left(\mathcal{G}^{\prime}(P), \Theta_{k} ; e^{1}\right)$, where $\left(\mathcal{G}^{\prime}(P), \Theta_{k}\right)$ is the graph of groups depicted below with

$$
\Gamma_{P^{j}}=\operatorname{im}\left(\pi_{1}\left(P^{j}, p^{j}\right) \stackrel{i_{*}}{\longrightarrow} \pi_{1}\left(M^{n}, p^{j}\right) \stackrel{\widehat{\gamma^{j}}}{\longrightarrow} \pi_{1}\left(M^{n}, p^{1}\right)\right)
$$

and $\gamma^{j}=\alpha^{j} * \beta^{j} *\left(\alpha^{1}\right)^{-1}$

(2) $\pi_{1}\left(W\left(Q, m^{\prime}\right),\left(q^{1}, 0\right)\right) \cong \pi_{1}\left(\mathcal{G}^{\prime}(Q), \Theta_{k} ; e^{1}\right)$ (with $\left(\mathcal{G}^{\prime}(Q), \Theta_{k}\right)$ analogous to the figure below), where

$$
\Gamma_{Q^{j}}=\operatorname{im}\left(\pi_{1}\left(Q^{j}, q^{j}\right) \stackrel{i_{*}}{\longrightarrow} \pi_{1}\left(M^{n}, q^{j}\right) \stackrel{\widehat{\beta^{j}}}{\longrightarrow} \pi_{1}\left(M^{n}, q^{1}\right)\right),
$$

(3) the homomorphism $\lambda$ is surjective,

(4) if there exists a homotopy pulling $M^{n}$ into $P$, then $\lambda$ is an isomorphism.

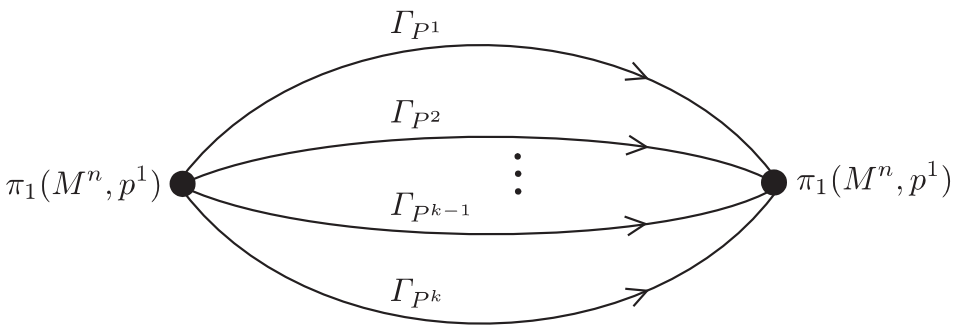

$\left(\mathcal{G}^{\prime}(P), \Theta_{k}\right)$ 
Proof. As noted in the comments preceding this proposition, (1) and (2) are essentially just applications of the generalized Seifert-van Kampen theorem. Assertion (3) is valid for nearly the same reason as assertion (3) of Proposition 3.5; in this case, $\lambda: \pi_{1}\left(\mathcal{G}^{\prime}(Q), \Theta_{k} ; e^{1}\right) \rightarrow \pi_{1}\left(\mathcal{G}^{\prime}(P), \Theta_{k} ; e^{1}\right)$ is induced by the natural isomorphism

$$
\pi_{1}\left(M^{n}, q\right) * \pi_{1}\left(M^{n}, q\right) * F_{E} \rightarrow \pi_{1}\left(M^{n}, p\right) * \pi_{1}\left(M^{n}, p\right) * F_{E},
$$

where $F_{E}$ is the free group on generators $E=\left\{e^{1}, \ldots, e^{k}\right\}$, by taking appropriate quotients in the domain and the range (as prescribed by the definition of the fundamental group of a graph of groups). If there exists a homotopy pulling $M^{n}$ into $P$, Corollary 3.3 makes it clear that this homomorphism is an isomorphism.

Corollary 3.8. Let $M^{n}$ be a connected, $k$-ended open $n$-manifold. Then $M^{n} \times \mathbb{R}$ is a one-ended open $(n+1)$-manifold with semistable fundamental group at infinity. If $M^{n}$ is finitely dominated, then $M^{n} \times \mathbb{R}$ has stable fundamental group at infinity which is pro-isomorphic to the system

$$
\pi_{1}\left(\mathcal{G}^{\prime}, \Theta_{k} ; e^{1}\right) \stackrel{\text { id }}{\longleftarrow} \pi_{1}\left(\mathcal{G}^{\prime}, \Theta_{k} ; e^{1}\right) \stackrel{\text { id }}{\longleftarrow} \pi_{1}\left(\mathcal{G}^{\prime}, \Theta_{k} ; e^{1}\right) \stackrel{\text { id }}{\longleftarrow} \cdots,
$$

where $\left(\mathcal{G}^{\prime}, \Theta_{k}\right)$ is the graph of groups pictured below. Here each $\Gamma_{j}$ is the image - translated by an appropriate change of basepoint isomorphism-of the fundamental group of the jth component of any sufficiently small 0neighborhood of infinity in $\pi_{1}\left(M^{n}\right)$, and the edge homomorphisms are all inclusions.

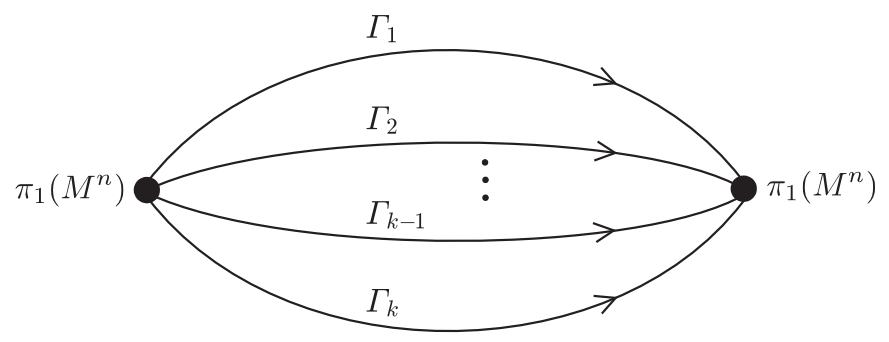

$\left(\mathcal{G}^{\prime}, \Theta_{k}\right)$

Proof. Choose a well-indexed neat sequence $\left\{N_{i}\right\}_{i=1}^{\infty}$ of 0 -neighborhoods of infinity in $M^{n}$. Then apply the above proposition repeatedly to the sequence $\left\{W\left(N_{i}, i\right)\right\}_{i=1}^{\infty}$ of associated neighborhoods of infinity in $M^{n} \times \mathbb{R}$. If $M^{n}$ is finitely dominated, $N_{1}$ should be chosen sufficiently small that $M^{n}$ can be pulled into $M^{n}-N_{1}$.

4. Closing comments. As indicated in the "easy part" of Theorem 1.1, if $M^{n}$ is a finitely dominated open manifold that is not homotopy equivalent to a finite complex, then $M^{n} \times \mathbb{R}$ is not compactifiable by the addition of a 
manifold boundary. By comparing Propositions 3.1 and 3.2 with Theorem 2.3 , it must be the case that $\sigma_{\infty}\left(M^{n} \times \mathbb{R}\right)$ is nontrivial. Since $M^{n}$ does not have finite homotopy type, its Wall obstruction $\sigma\left(M^{n}\right)$ is a nontrivial element of $\widetilde{K}_{0}\left(\mathbb{Z}\left[\pi_{1}\left(M^{n}\right)\right]\right)$. As one might expect, there is a relationship between $\sigma_{\infty}\left(M^{n} \times \mathbb{R}\right)$ and $\sigma\left(M^{n}\right)$.

By Proposition 3.7 and Corollary 3.8, $\sigma_{\infty}\left(M^{n} \times \mathbb{R}\right)$ may be viewed as the Wall finiteness obstruction of $W\left(M^{n}, N\right)$, where $N$ is any sufficiently small 0-neighborhood of infinity in $M^{n}$; this obstruction $\sigma\left(W\left(M^{n}, N\right)\right.$ ) lies in $\widetilde{K}_{0}\left(\mathbb{Z}\left[\pi_{1}(W(N, m))\right]\right)$. The retraction of $W(N, m)$ onto $M^{n} \times\{m\}$ obtained by projection shows that $\pi_{1}\left(M^{n}\right)$ is a retraction of $\pi_{1}(W(N, m))$; so, by functoriality, $\widetilde{K}_{0}\left(\mathbb{Z}\left[\pi_{1}\left(M^{n}\right)\right]\right)$ is a retraction of $\widetilde{K}_{0}\left(\mathbb{Z}\left[\pi_{1}(W(N, m))\right]\right)$. As a consequence, the inclusion induced homomorphism $i_{*}: \widetilde{K}_{0}\left(\mathbb{Z}\left[\pi_{1}\left(M^{n}\right)\right]\right) \rightarrow$ $\widetilde{K}_{0}\left(\mathbb{Z}\left[\pi_{1}(W(N, m))\right]\right)$ is injective. By applying the sum theorem for Wall's finiteness obstruction [Si1, Ch. VI] to the homotopy equivalence $W(N, m) \simeq$ $K_{N}=M^{n} \cup_{\varphi} C^{\prime}$ utilized in the proof of Proposition 3.1, it is easy to see that $\sigma\left(W\left(M^{n}, N\right)\right)$ is precisely $i_{*}\left(\sigma\left(M^{n}\right)\right)$.

As a consequence of all of the above, we have a recipe for creating open manifolds that are reasonably nice at infinity (inward tame with stable fundamental group), but are not compactifiable via the addition of a manifold boundary. Specifically: build a finite-dimensional complex $K$ that is finitely dominated but $\sigma(K) \neq 0$; properly embed $K$ in $\mathbb{R}^{n}$ and let $M^{n}$ be the interior of a proper regular neighborhood of $K$; then $M^{n} \times \mathbb{R}$ satisfies conditions (1) and (2) of Theorem 2.3, but $\sigma_{\infty}\left(M^{n} \times \mathbb{R}\right)$ is nontrivial and equal to $i_{*}(\sigma(K))$.

\section{References}

[Co] D. E. Cohen, Combinatorial Group Theory: A Topological Approach, London Math. Soc. Student Texts 14, Cambridge Univ. Press, Cambridge, 1989.

[Fr] M. H. Freedman, The topology of four-dimensional manifolds, J. Differential Geom. 17 (1982), 357-453.

[Ge] R. Geoghegan, Topological Methods in Group Theory, in preparation.

[Gl] J. Glimm, Two Cartesian products which are Euclidean spaces, Bull. Soc. Math. France 88 (1960), 131-135.

[Gu] C. R. Guilbault, Manifolds with non-stable fundamental groups at infinity, Geom. Topol. 4 (2000), 537-579.

[GuTi] C. R. Guilbault and F. C. Tinsley, Manifolds with non-stable fundamental groups at infinity, II, ibid. 7 (2003), 255-286.

[Hu] S. T. Hu, Theory of Retracts, Wayne State Univ. Press, Detroit, MI, 1965.

[KS] R. C. Kirby and L. C. Siebenmann, Foundational Essays on Topological Manifolds, Smoothings, and Triangulations, Ann. of Math. Stud. 88, Princeton Univ. Press, Princeton, NJ, 1977.

[Lu1] E. Luft, On contractible open topological manifolds, Invent. Math. 4 (1967), 192201. 
[Lu2] E. Luft, On contractible open 3-manifolds, Aequationes Math. 34 (1987), 231239.

[Ma] W. S. Massey, Algebraic Topology: An Introduction, Grad. Texts in Math. 56, Springer, New York, 1977.

[Mc] D. R. McMillan, Jr., Cartesian products of contractible open manifolds, Bull. Amer. Math. Soc. 67 (1961), 510-514.

[Si1] L. C. Siebenmann, The obstruction to finding a boundary for an open manifold of dimension greater than five, Ph.D. thesis, Princeton Univ., 1965.

[Si2] - On detecting open collars, Trans. Amer. Math. Soc. 142 (1969), 201-227.

[St] J. Stallings, The piecewise-linear structure of Euclidean space, Proc. Cambridge Philos. Soc. 58 (1962), 481-488.

[Wa] C. T. C. Wall, Finiteness conditions for CW-complexes, Ann. of Math. 81 (1965), $56-69$.

[Wh] J. H. C. Whitehead, A certain open manifold whose group is unity, Quart. J. Math. Oxford Ser. 6 (1935), 268-279.

Department of Mathematical Sciences

University of Wisconsin-Milwaukee

Milwaukee, WI 53201, U.S.A.

E-mail: craigg@uwm.edu

Received 22 December 2005;

in revised form 20 March 2007 